\title{
Leukocytospermia in late adolescents: possible clinical interpretations
}

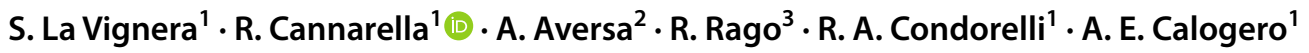

Received: 6 June 2020 / Accepted: 28 October 2020 / Published online: 23 November 2020

(c) The Author(s) 2020

\begin{abstract}
Background No data are currently available on the implication of amicrobial leukocytospermia in male adolescents. Therefore, the primary aim of this study was to evaluate the prevalence of amicrobial leukocytospermia among non-smoker late adolescents who were exposed to other risky lifestyles for the andrological health. The main andrological clinical features of adolescents with leukocytospermia were also reported.

Methods This is a cross-sectional study carried out in 80 boys. Each adolescent underwent a physical examination, and to the assessment of sperm conventional parameters, seminal leukocytes concentration and immature germ cell evaluation. A possible correlation between seminal leukocytes and immature germ cells and testicular volume (TV) was tested.

Results The adolescents enrolled in this study had 18.0 0.4 (range 18.1-18.9) years. Unprotected sexual intercourse was referred by $38 \%$ of them. Sexual dysfunctions were found in $25 \%$ and isolated hypoactive sexual desire in $12.5 \%$ of boys. Low TV and penile length in flaccidity were found in $44 \%$ and $30 \%$ of them, respectively. Only $41 \%$ had normozoospermia at the sperm analysis, whereas $19 \%$ had isolated oligozoospermia, $15 \%$ oligo-asthenozoospermia, and $25 \%$ oligo-asthenoteratozoospermia. Leukocytospermia occurred in 25\% (20 out of 80 ) of adolescents. No seminal infection was detected in $19 \%$ (15 out of 80 ) of them. Adolescents with leukocytospermia had lower progressive sperm motility, percentage of normal forms, TV, and a higher percentage of immature germ cells compared to those without leukocytospermia. Semen leukocyte concentration correlated negatively with TV and positively with the percentage of immature germ cells in the ejaculate.

Conclusion Leukocytospermia, increased immature germ cell number, and low TV identify a distinct phenotype suggestive of testicular tubulopathy. Primary prevention of male infertility and the counselling for andrological risky lifestyles is mandatory and should be started as early as possible.
\end{abstract}

Keywords Testicular volume $\cdot$ Male infertility $\cdot$ Primary prevention $\cdot$ Leukocytospermia $\cdot$ Immature germ cells $\cdot$ Oligozoospermia

\section{Introduction}

There is much debate about when the first sperm analysis should be requested, although evidence suggests no earlier than a year and a half after the onset of puberty [1]. Conventionally, the steps preceding full sexual maturation include

S. La Vignera

sandrolavignera@unict.it

1 Department of Clinical and Experimental Medicine, University of Catania, Policlinico "G. Rodolico", via S. Sofia 78, 95123 Catania, Italy

2 Department of Experimental and Clinical Medicine, "Magna Graecia" University, 88100 Catanzaro, Italy

3 Physiopathology of Reproduction and Andrology Unit, Sandro Pertini Hospital, 00157 Rome, Italy spermaturia (the presence of spermatozoa in the urine) and ejacularche (the first conscious ejaculation) [1]. In addition to the sperm analysis, a testicular volume (TV) lower than that normally expected for the Tanner stage, the maintenance of high serum levels of anti-Müllerian hormone (AMH), and low inhibin $B$ values may be additional useful criteria to carefully monitor the transitional age during puberty [2-4].

Sperm analysis should be performed by a fully-trained and experienced seminologist engaged in a quality control program and interpreted by a trained clinician. The presence of abnormal conventional sperm parameters (concentration, motility, and morphology) results in a diagnosis of oligozoospermia (sperm count $<15 \times 10^{6} / \mathrm{ml}$ and total sperm count $<39$ million/ejaculate), asthenozoospermia (progressive sperm motility $<32 \%$ and total motility $<40 \%$ ), and/ or teratozoospermia (normal forms $<4 \%$ ) [5]. A second 
sperm analysis is, however, mandatory because of the elevated intra-individual variations also in men with normal $\mathrm{TV}$ and/or normal serum levels of gonadotropins, inhibin $\mathrm{B}$ and $\mathrm{AMH}$.

The association between varicocele and abnormal sperm parameters is well known in the adulthood [6]. By contrast, few evidence is available on this matter in the adolescence [3], when testicular asymmetry, scrotal pain, and/or hormone abnormalities suggest the correction of varicocele [7, 8]. Several other diseases are able to affect sperm parameters, such as genetic disorders (karyotype abnormalities, numeric or structural chromosome alterations, Y chromosome microdeletions, syndromic genetic disorders, single gene mutations) $[9,10]$, male accessory gland infection or inflammation [11], congenital or acquired hypogonadism, testicular tumor, testicular torsion, chemotherapy, and radiotherapy. Importantly, pre-natal exposure to endocrine disruptors or maternal cigarette smoke can similarly damage spermatogenesis in the adulthood [12]. Furthermore, cigarette smoke, marijuana, alcohol intake, obesity during adolescence can also negatively impact on spermatogenesis [13-15].

The finding of an increase in the concentration of leukocytes in the semen in adolescents is still matter of debate. However, the close relationship between leukocytospermia $(>1 \mathrm{million} / \mathrm{ml}$ ) and urogenital infections is widely accepted and, therefore, the microbiological assessment is currently suggested in these patients [17]. A low leukocyte concentration, by causing a limited sperm exposure to reactive oxygen species, associates with a better sperm quality [18-20]. Based on these premises, the study aims were twofold. First, to evaluate the prevalence of leukocytospermia in non-smoker, healthy (with no major andrological or general comorbidities) late adolescents, enrolled in an andrology screening program carried out in the city of Catania (Italy). The second purpose was to assess the main andrological clinical characteristics of adolescents with leukocytospermia.

\section{Patients and methods}

\section{Patients}

Eighty adolescents of $18 \pm 0.4$ years old (range 18.1-18.9 years) agreed to undergo semen analysis as part of an andrological screening program conducted in the city of Catania (Southern Italy) in the last 3 years.

The clinical evaluation consisted in the collection of the andrological medical history aimed at assessing the presence of risk factors for male infertility (Table 1) and the physical examination for the assessment of the following parameters: TV; length of the penis in flaccidity; presence
Table 1 Risk factors for infertility found in 80 healthy 18 -years-old adolescents

\begin{tabular}{lrr}
\hline Risk factor & $n$ & $\%$ \\
\hline Sexual promiscuity & 11 & 14 \\
Drug abuse & 8 & 10 \\
Alcohol abuse* & 15 & 19 \\
Testicular trauma & 5 & 6 \\
Anabolic-androgenic steroids & 3 & 4 \\
\hline
\end{tabular}

The risk factors not included in this table were considered exclusion criteria

$*>36$ g/day

of gynecomastia; presence of genital warts; presence of abnormalities of the urethral meatus. They all were in Tanner Stage V.

At enrollment the following exclusion criteria were adopted: overweight/obesity [body mass index $(\mathrm{BMI})>25 \mathrm{~kg} / \mathrm{m}^{2}$ ], chemotherapy, radiotherapy, endocrine diseases, varicocele (from the first to the third stage according to the Dubin's classification), cryptorchidism, testicular tumor, cigarette smoke, previous urogenital surgery, urogenital infection in pediatric and adolescent age, orchitis, genetic disorders, use of any medication for other health reasons, presence of sperm antibodies.

\section{Semen analysis}

Semen samples were collected from each adolescent after 4 days of sexual abstinence. The semen analysis was performed following the criteria suggested by the World Health Organization manual [5]. Each specimen was analyzed by two operators for quality control. Semen samples with a leukocyte concentration $>1 \mathrm{million} / \mathrm{ml}$ were sent to the microbiology laboratory, and the following microorganisms were searched: Escherichia coli, Enterococcus faecalis, Klebsiella, Mycoplasma hominis, Ureaplasma urealyticum, Chlamydia trachomatis, Trichomonas vaginalis, Gardnerella vaginalis, Candida.

\section{Seminal leukocyte evaluation (peroxidase test)}

The protocol used was adapted from that of Endtz [21]. The working solution was obtained by adding $1 \mu \mathrm{l}$ of $\mathrm{H}_{2} \mathrm{O}_{2}$ to $20 \mu \mathrm{l}$ of a $0.09 \%$ 3,3'-diaminobenzidine tetrahydrochloride stock solution (DAB, ISOPAC, Sigma, Milan, Italy) in $40 \%$ ethanol. $20 \mu \mathrm{l}$ of semen were incubated with $20 \mu \mathrm{l}$ of working solution for $5 \mathrm{~min}$ at room temperature in each assay. Before setting up the slide, $40 \mu 1$ of PBS were added. Peroxidase-positive cells were marked by yellow-brown-red staining, while peroxidase-negative cells remained colorless. At least 100 round cells were counted using an optical 
microscope at $400 \times$ magnification and the percentages of peroxidase-positive and -negative cells were evaluated. The total leukocyte count has been expressed in millions per milliliter of semen.

\section{Evaluation of immature germ cells}

Spermatids were differentiated from leukocytes by a semen smear stained using the Papanicolaou technique [22]. Spermatids were identified on the basis of the following parameters: coloration, size, core shape and size (approximately $5 \mu \mathrm{M}$ ), absence of intracellular peroxidase and the absence of leukocyte-specific antigen. Morphologically, multinucleated spermatids were distinguished from polymorphonuclear leukocytes by the presence of a pink color in contrast to the bluish color of polymorphonuclear leukocytes.

\section{Mixed antiglobulin reaction (MAR) test}

All samples were analyzed for the presence of sperm antibodies using the MAR test. The MAR test was performed according with the criteria suggested by the WHO 2010 manual [5]. Briefly, two aliquots of $3.5 \mu \mathrm{l}$ of semen were prepared and placed on separate microscope slides. One slide was then incubated with $3.5 \mu \mathrm{l}$ of antibody-positive semen and one with $3.5 \mu \mathrm{l}$ of antibody-negative semen as control. An aliquot of $3.5 \mu \mathrm{l}$ of IgG-coated latex particles (beads) was added to each droplet of test and control semen, and properly mixed up by stirring with the pipette tip. An aliquot of $3.5 \mu \mathrm{l}$ of antiserum against human IgG was added to each semen-bead mixture and mixed-up by stirring with a pipette tip. After covering, the slides were stored horizontally for $3 \mathrm{~min}$ at room temperature in a humidified chamber. The methodology was similar for IgA but IgA-coated beads and anti-IgA antibodies were used. The slides were observed at microscope at $\times 200$ magnification after 3 min and again after $10 \mathrm{~min}$, to assess the percentage of motile spermatozoa carrying beads. As the WHO 2010 manual indicates [5], 50\% of motile spermatozoa with adherent particles was considered as a threshold value.

\section{Scrotal ultrasound evaluation}

The ultrasound examination was performed with a GX Megas Esaote (Esaote SpA, Genoa, Italy) device, equipped with linear, high-resolution, and high-frequency ( 7.5 to $14 \mathrm{MHz}$ ) probes dedicated to the study of soft body areas, with color Doppler for detecting slow flow and a scanning surface of at least $5 \mathrm{~cm}$. The TV was calculated using the ellipsoid formula (length $\times$ width $\times$ thickness $\times 0.52$ ). The testis was considered hypotrophic when it had a volume of less than $12 \mathrm{~cm}^{3}$ [23, 24]. TV was evaluated as the mean of the volumes of the right and left testes.

\section{Statistical analysis}

Results are reported as mean $\pm \mathrm{SD}$ and in percentages throughout the study. The normal distribution of the variables was evaluated with the Shapiro-Wilk test. The association of leukocytospermia with TV and immature germ cells was analyzed using the Pearson correlation or Spearman rank correlation according to the normal distribution of the variables and the results are reported as correlation coefficient $(r)$ and $p$ value. Data collected from patients with and without leukocytospermia were analyzed by Student's $t$ test. Statistical analysis was performed using SPSS 22.0 for Windows (SPSS Inc., Chicago, IL, USA). A $p$ value lower than 0.05 was accepted as statistically significant.

\section{Ethical approval}

This study was carried out at the Division of Andrology and Endocrinology of the University Teaching hospital "G. Rodolico", University of Catania (Catania, Italy). The protocol was approved by the internal Institutional Review Board and an informed written consent was obtained from each participant after full explanation of the purpose and nature of all procedures used. The study has been conducted in accordance with the principles expressed in the Declaration of Helsinki.

\section{Results}

The medical history showed that 30 out of 80 (38\%) adolescent reported promiscuous sexual activity without contraceptive methods, 20/80 (25\%) of them referred isolated erectile dysfunction and/or premature ejaculation during sexual activity, 10/80 (12.5\%) reported the presence of isolated hypoactive sexual desire. 35/80 (44\%) adolescents showed low TV ( $<12 \mathrm{ml}) ; 15 / 80$ (30\%) low mean penile length in flaccidity using a cut-off of $9.3 \mathrm{~cm}$, equivalent to -2.5 standard deviations of the normal penile length of an adult man [13] (Table 2).

Overall, the mean sperm concentration was $40 \pm 33$ million $/ \mathrm{ml}$, the mean value of progressive motility was $30 \pm 18 \%$, and the mean value of typical forms was $8 \pm 6 \%$. Only $33 / 80(41 \%)$ boys showed normozoospermia at the semen analysis; 15/80 (19\%) isolated oligozoospermia, $12 / 80$ (15\%) oligo-asthenozoospermia and 20/80 (25\%) oligo-astheno-teratozoospermia.

Notably, 20/80 (25\%) adolescents had increased seminal leukocyte concentration ( $>1$ million/ml). Hence, based on the presence or the absence of leukocytospermia $(>1$ million/ml), two groups of patients were identified: those with $(2.6 \pm 1.1 \mathrm{million} / \mathrm{ml})$ and without $(0.6 \pm 0.3 \mathrm{mil}-$ lion/ml) leukocytospermia $(p<0.01)$. The consequent 
Table 2 Testicular volume (TV) and penile length in 80 healthy 18-years-old adolescents

\begin{tabular}{lll}
\hline Parameter & $\begin{array}{l}\text { Mean value } \\
( \pm \mathrm{SD})\end{array}$ & $\begin{array}{l}\text { Percentage of adoles- } \\
\text { cents with abnormal } \\
\text { values }\end{array}$ \\
\hline Right TV $(\mathrm{ml})$ & $17 \pm 8$ & 40 \\
Left TV $(\mathrm{ml})$ & $17 \pm 6$ & 48 \\
Mean TV (ml) & $16 \pm 7$ & 44 \\
$\begin{array}{l}\text { Stretched penile length } \\
(\mathrm{cm})\end{array}$ & $12 \pm 6$ & 30 \\
\hline
\end{tabular}

Normal value for $\mathrm{TV}: \geq 12 \mathrm{ml}$; normal value for stretched penile length: $\geq 9.3 \mathrm{~cm}$. Varicocele was an exclusion criterion

microbiological assessment revealed the presence of infection in only $5 / 20$ cases. The remaining 15 adolescents with leukocytospermia had no microbial infection in their semen. Progressive sperm motility (Fig. 1, a) and normal forms (Fig. 1, b) resulted significantly lower than those found in adolescents without leukocytospermia. The percentage of spermatids (Fig. 1, c) in the semen was significantly higher. Particularly, the group of patients with leukocytospermia had $16 \pm 4$ spermatids over 100 spermatozoa, whereas the group without leukocytospermia had $5 \pm 2$ spermatids over 100 spermatozoa $(p<0.01)$. By contrast, sperm concentration $(30 \pm 23 \mathrm{million} / \mathrm{ml}$ vs. $43 \pm 18 \mathrm{million} / \mathrm{ml})$ did not differ significantly in the two groups of adolescents. Finally, adolescents with leukocytospermia had a significantly lower testicular volume (Fig. 2). Testicular hypotrophy was found in 12/20 adolescents with leukocytospermia. No adolescent with testicular hypotrophy was found in the group without leukocytospermia.

The correlation analysis showed a significant inverse relationship between leukocyte concentration and TV $(r=-0.83 ; p<0.05)$ and the percentage of spermatids $(r=0.87 ; p<0.05)$.

\section{Discussion}

The results of the present study showed the presence of abnormal sperm parameters in many healthy late adolescents. The alteration of at least one of the main conventional sperm parameters (sperm concentration, progressive motility or morphology) was found in 59\% of them. Leukocytospermia was found in $25 \%$ of them and resulted idiopathic in the vast majority of them (75\%), being the microbiological tests negative for bacterial and fungal infection and the didymo-epididymal ultrasound negative for ultrasound signs of inflammation. In addition, adolescents with leukocytospermia showed a lower sperm progressive motility, percentage of normal forms, TV, and a higher percentage of spermatids compared to adolescents without leukocytospermia.
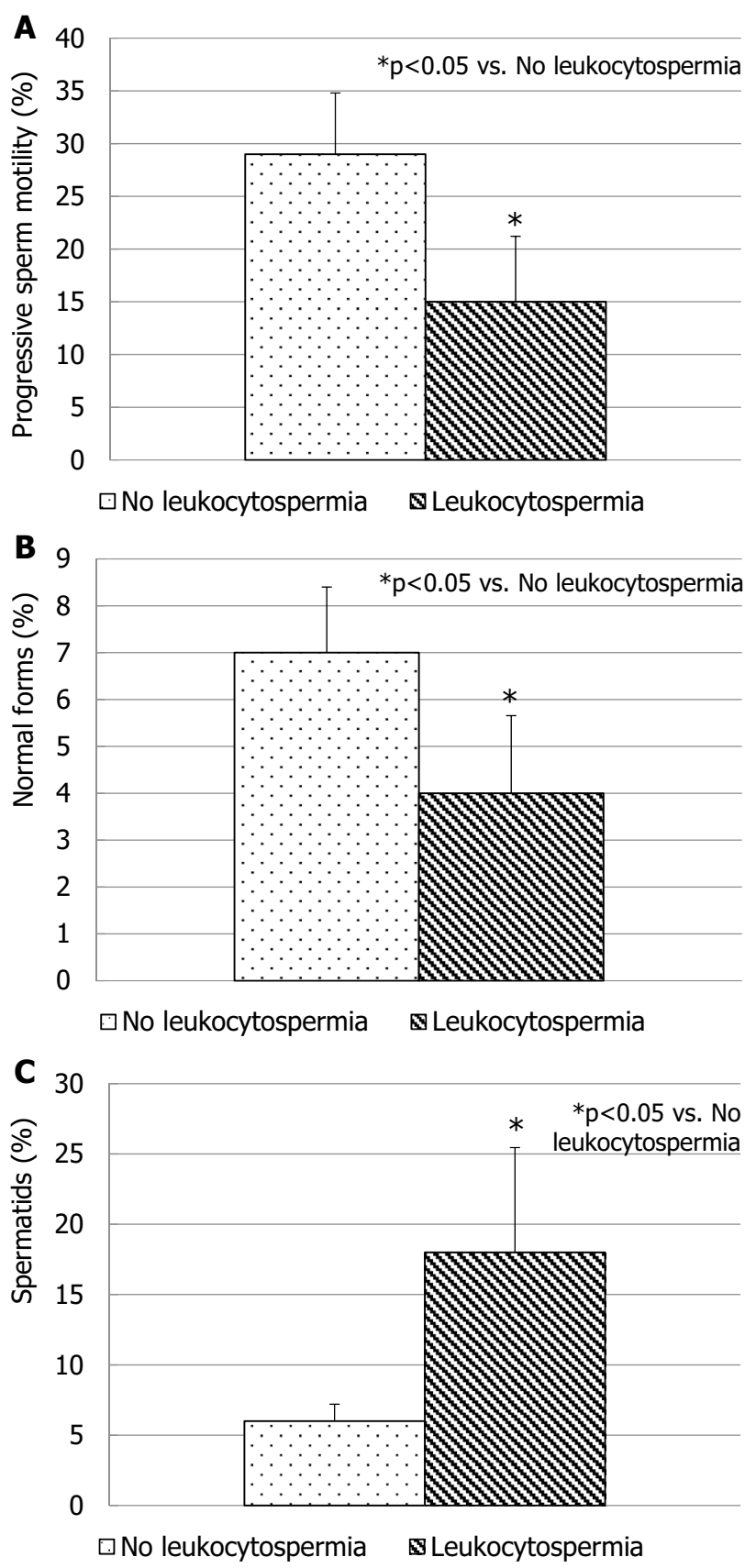

Fig. 1 Sperm progressive motility (a), morphology (b) and spermatid percentage (c) in patients with and without leukocytospermia. Standard deviation is indicated by bars

Furthermore, sperm leucocyte concentration correlated negatively with the TV and positively with the percentage of spermatids.

The interpretation of these findings are of great interest because important risk factors for male fertility, such as varicocele, male accessory gland infections, cigarette smoke, obesity, cryptorchidism, testicular tumor, previous urogenital surgery, genetic disorders, or use of any medication for 


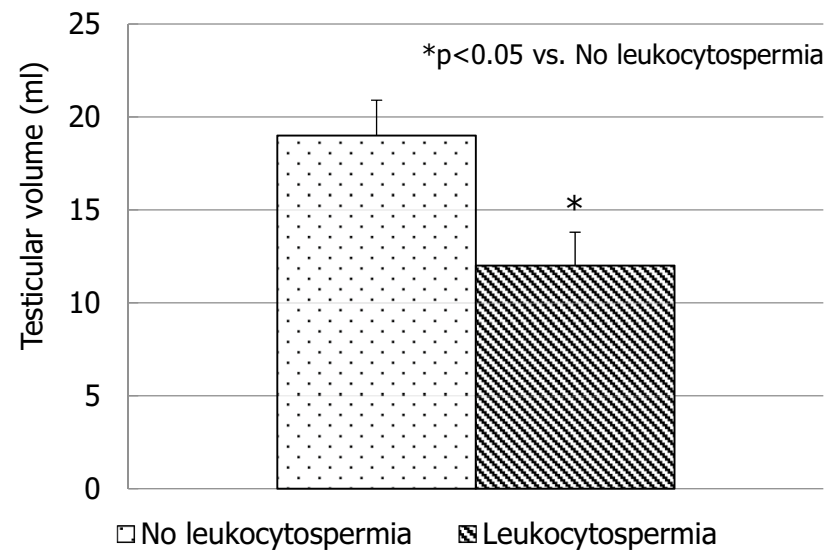

Fig. 2 Testicular volume in patients with and without leukocytospermia. Standard deviation is indicated by bars

other medical reasons, were taken into account and considered exclusion criteria. Other risk factors or risky lifestyles found in adolescents included in this study, such as drug or alcohol abuse, are listed in Table 1.

The presence of abnormal conventional sperm parameters at such a high frequency shows that primary prevention of male fertility should start very early in life and that the evaluation of testicular growth should be one of the parameters to be taken into consideration. In the event of abnormalities, measurements of serum AMH and inhibin B levels should be undertaken and risky lifestyle identified and adolescent properly counseled to eliminate them to preserve their reproductive health $[4,25]$.

There is large consensus in the literature for a decline of sperm concentration and total sperm number, which halved in the last forty years without any apparent explanation [26]. This has led to the development of studies that have evaluated sperm parameters in late adolescence, as well as to estimate the prevalence of risky lifestyle for the andrological health in this population, such as smoke, alcohol, drug consumption, unprotected sexual intercourse, which highlights the importance of undergoing to andrological counseling in adolescence. Thus, dysmetabolism and risky lifestyle negatively interfere with male gonadal function at a young age and deserve fully attention [13-16]. Accordingly, recent studies have shown the detrimental impact of lifestyle factors on testicular function in adolescence. A study including 1215 young Danish men aged 18-28 years reported a dosedependent decline in sperm concentration and total sperm count in marijuana smokers, which accounted for $45 \%$ of this cohort or youngsters [13]. In line with these findings, an Italian survey carried out in 10,124 18 years-old male students attending last year of the high school reported a not negligible prevalence of risky lifestyles for the andrological health, such as smoking (32.6\%), alcohol intake (80.6\%), and use of illegal drugs (46.5\%). Unprotected sexual intercourse was reported by $48.3 \%$ of these students and of their partners, thus increasing the risk to develop sexual transmitted diseases (STDs). Remarkably, up to $14 \%$ had bilateral testicular hypotrophy (TV $<12 \mathrm{ml}$ ) at the physical examination, which correlated positively with underweight and heavy alcohol or drug use [14]. Similarly, another Italian study conducted in 360 male high-school students reported an elevated prevalence of cigarette smoke (41.5\%), alcohol consumption (9.3\%), and cannabis use (19.7\%). Their overall awareness about the risk of STDs was low [15]. Finally, a longitudinal study prospectively evaluated the presence of correlations between insulin-resistance and non-alcoholic fatty liver disease (NAFLD) with TV and sperm parameters. Interestingly, this study showed a 50\% decrease in sperm output and a TV lower by $\sim 20 \%$ at the age of 20 in adolescents with NAFLD and insulin-resistance when they were 17 year-old [16].

This evidence supports the findings of the present study. Lifestyles (Table 1) who adolescents were exposed to are potentially able to negatively impact of testicular function. However, the abnormality of mean TV, conventional sperm parameters, and penile length were found in a nonnegligible prevalence and the presence of other unknown factors impacting on these outcomes cannot be excluded. Importantly, idiopathic leukocytospermia occurred in 12/80 patients. These adolescents had worse sperm parameters (lower sperm motility and morphology), significantly lower TV and higher spermatid concentration compared to adolescents without leukocytospermia. This group of adolescent may be at risk of a testicular damage during youth, which may predispose them to a decreased sperm output later in life. Furthermore, to our knowledge, this is the first report of idiopathic (amicrobial) leukocytospermia in adolescents and, therefore, its interpretation/significance is not fully codified.

Leukocytospermia is an established marker of inflammation and it has been associated with increased semen levels of interleukin- 6 and tumor necrosis factor- $\alpha$, both inflammatory cytokines, in infertile patients [27]. Leukocytes are also involved in the pathogenesis of autoimmune inflammation of seminal ducts [28]. The blood-testis barrier (BTB) is made by Sertoli cells to defend auto-immunogenic spermatids from the self-immune system attack. However, some penetrating lymphocytes lie very close to the germ cells, being retrieved in mice tubuli recti, rete testis and epididymis, but not in the seminiferous tubules, under normal conditions. These cells have been advocated to the pathogenesis of autoimmune inflammation of tubuli recti, rete testis and epididymis [28]. The disruption of BTB integrity is involved in the pathogenesis of worse seminal phenotypes, such as Sertoli cell only syndrome or hypospermatogenesis [29]. The BTB, which makes the testis an immunologically privileged organ, literary divides the epithelium of seminiferous tubules into two compartments: the basal one, including 
germ cell in I/II meiosis and the adluminal one, where post-meiotic spermatids and spermatozoa can be retrieved [30]. The increase in immature germ cells in the semen, including spermatocytes and spermatids, is indicative for testicular damage, as the WHO manual indicates [5]. The higher leukocyte and spermatid percentage reported in the adolescents included in the present study may indicate the presence of a testicular tubulopathy, which is strongly supported by the presence of a decreased TV. TV represents a widely accepted marker of testicular function since it correlates positively with conventional sperm parameters [31]. While in the pre-pubertal phase TV reflects Sertoli cell proliferation, it is related with the amount of spermatogenesis in testicular tubules during the post-pubertal phase [2]. Hence, the concomitant presence of a reduced TV supports the hypothesis of tubular dysfunction. However, longitudinal studies are warranted to confirm this hypothesis.

We are aware of some important study limitations. First of all, the cross-sectional nature of the study does not allow any conclusive data regarding these young boys and their possibility to recover sperm parameters naturally. Second, we did not perform HPV analysis as a possible cause of leukocytospermia and we are aware that its incidence amongst young boys who have unprotected sex is quite high. In addition, sperm culture was the only microbiological test which patients underwent to. This may have underestimated the real prevalence of bacterial infection. Last but not least, the impossibility to investigate any organic cause of erectile dysfunction and correlation with sexual hormones as well as with penile duplex ultrasound [31].

\section{Conclusion}

In conclusion, we have found the presence of alteration of at least one of the conventional sperm parameters in more than a half of healthy adolescent men. Furthermore, a distinct phenotype characterized by leukocytospermia, increased spermatid concentration and low TV was identified, possibly indicating the presence of testicular tubulopathy. We have also found a non-negligible prevalence of testicular hypotrophy and low penile growth. In line with previous findings, our data strongly highlight the importance of primary prevention of male infertility, which should be accomplished starting from pre-pubertal age by monitoring testicular growth, measuring AMH and inhibin B serum levels in selected cases $^{31}$ and counselling boys and adolescents for identifying/eliminating risky lifestyles for andrological health.

Funding Open access funding provided by Università degli Studi di Catania within the CRUI-CARE Agreement.. This research did not receive any specific grant from funding agencies in the public, commercial, or not-for-profit sectors.

\section{Compliance with ethical standards}

Conflict of interest The authors declare that there is no conflict of interest that could be perceived as prejudicing the impartiality of the research reported.

Ethical approval The study was conducted in accordance with the principles expressed in the Declaration of Helsinki. The protocol was approved by the Internal Review Board of the Division of Andrology and Endocrinology, University of Catania, Catania, Italy.

Informed consent Informed written consent was obtained from each participant after full explanation of the purpose and nature of all procedures used.

Open Access This article is licensed under a Creative Commons Attribution 4.0 International License, which permits use, sharing, adaptation, distribution and reproduction in any medium or format, as long as you give appropriate credit to the original author(s) and the source, provide a link to the Creative Commons licence, and indicate if changes were made. The images or other third party material in this article are included in the article's Creative Commons licence, unless indicated otherwise in a credit line to the material. If material is not included in the article's Creative Commons licence and your intended use is not permitted by statutory regulation or exceeds the permitted use, you will need to obtain permission directly from the copyright holder. To view a copy of this licence, visit http://creativecommons.org/licenses/by/4.0/.

\section{References}

1. Dabaja AA, Wosnitzer MS, Bolyakov A, Schlegel PN, Paduch DA (2014) When to ask male adolescents to provide semen sample for fertility preservation? Transl Androl Urol 3:2-8

2. Condorelli RA, Cannarella R, Calogero AE, La Vignera S (2018) Evaluation of testicular function in prepubertal children. Endocrine 62:274-280

3. Edelsztein NY, Grinspon RP, Schteingart HF, Rey RA (2016) Anti-Müllerian hormone as a marker of steroid and gonadotropin action in the testis of children and adolescents with disorders of the gonadal axis. Int J Pediatr Endocrino 2016:20

4. La Vignera S, Condorelli RA, Cimino L, Cannarella R, Giacone F, Calogero AE (2019) Early identification of isolated Sertoli cell dysfunction in prepubertal and transition age: is it time? J Clin Med 8(5):636

5. World Health Organization (2010) WHO laboratory manual for the examination and processing of human semen, 5th edn. Cambridge University Press, Cambridge

6. Pallotti F, Paoli D, Carlini T, Vestri AR, Martino G, Lenzi A, Lombardo F (2018) Varicocele and semen quality: a retrospective case-control study of 4230 patients from a single centre. J Endocrinol Invest 41(2): 185-192

7. Locke JA, Noparast M, Afshar K (2017) Treatment of varicocele in children and adolescents: a systematic review and meta-analysis of randomized controlled trials. J Pediatr Urol 13(5):437-445

8. Cannarella R, Calogero AE, Condorelli RA, Giacone F, Aversa A, La Vignera S (2019) Management and treatment of varicocele in children and adolescents: an endocrinologic perspective. J Clin Med 8(9): 1410 
9. Cannarella R, Condorelli RA, Duca Y, La Vignera S, Calogero AE (2019) New insights into the genetics of spermatogenic failure: a review of the literature. Hum Genet 138(2):125-140

10. Asero P, Calogero AE, Condorelli RA, Mongioi' L, Vicari E, Lanzafame F, Crisci R, La Vignera S (2014) Relevance of genetic investigation in male infertility. J Endocrinol Invest 37(5):415-427

11. Duca Y, Calogero AE, Cannarella R, Condorelli RA, La Vignera S (2019) Current and emerging medical therapeutic agents for idiopathic male infertility. Expert Opin Pharmacother 20(1):55-67

12. Barbagallo F, Condorelli RA, Mongioì LM, Cannarella R, Aversa A, Calogero AE, La Vignera S (2020) Effects of bisphenols on testicular steroidogenesis. Front Endocrinol (Lausanne). 11:373

13. Gundersen TD, Jørgensen N, Andersson AM, Bang AK, Nordkap L, Skakkebæk NE, Priskorn L, Juul A, Jensen TK (2015) Association between use of marijuana and male reproductive hormones and semen quality: a study among 1215 healthy young men. Am J Epidemiol 182(6):473-481

14. Gianfrilli D, Ferlin A, Isidori AM, Garolla A, Maggi M, Pivonello R, Santi D, Sansone A, Balercia G, Granata ARM, Sinisi A, Lanfranco F, Pasqualetti P, Foresta C, Lenzi A, Andrologo' Study Group (2019) Risk behaviours and alcohol in adolescence are negatively associated with testicular volume: results from the Amico-Andrologo survey. Andrology 7(6):769-777

15. Perri A, Lofaro D, Izzo G, Aquino B, Bitonti M, Ciambrone G, La Vignera S, Pozza C, Gianfrilli D, Aversa A (2019) The risky health behaviours of male adolescents in the southern Italian region: implications for sexual and reproductive disease. J Clin Med 8(9): 1414

16. Hart RJ, Doherty DA, Mori TA, Adams LA, Huang RC, Minaee N, Handelsman DJ, McLachlan R, Norman RJ, Dickinson JE, Olynyk JK, Beilin LJ (2019) Features of the metabolic syndrome in late adolescence are associated with impaired testicular function at 20 years of age. Hum Reprod 34(3):389-402

17. Colpi GM, Francavilla S, Haidl G, Link K, Behre HM, Goulis DG, Krausz C, Giwercman A (2018) European Academy of Andrology guideline Management of oligo-astheno-teratozoospermia. Andrology 6(4):513-524

18. Fraczek M, Hryhorowicz M, Gill K, Zarzycka M, Gaczarzewicz D, Jedrzejczak P, Bilinska B, Piasecka M, Kurpisz M (2016) The effect of bacteriospermia and leukocytospermia on conventional and nonconventional semen parameters in healthy young normozoospermic males. J Reprod Immunol 118:18-27

19. Cannarella R, Condorelli RA, Mongioi' LM, Giacone F, Calogero AE, La Vignera S (2019) Management of male accessory gland inflammations: a response to Haidl et al. Andrologia 51(5):e13261
20. Lackner JE, Agarwal A, Mahfouz R, du Plessis SS, Schatzl G (2020) The association between leukocytes and sperm quality is concentration dependent. Reprod Biol Endocrinol 8:12

21. Endtz AW (1974) A rapid staining method for differentiating granulocytes from "germinal cells" in papanicolaou stained semen. Acta Cytol 18(1):2-7

22. Johanisson E, Campana A, Luthi R, de Agostini A (2000) Evaluation of 'round cells' in semen analysis: a comparative study. Hum Reprod Update 6(4):404-412

23. Sermondade N, Faure C, Fezeu L, Shayeb AG, Bonde JP et al (2013) BMI in relation to sperm count: an updated systematic review and collaborative meta-analysis. Hum Reprod Update 19(3):221-231

24. Levine H, Jørgensen N, Martino-Andrade A, Mendiola J, WekslerDerri D, Mindlis I, Pinotti R, Swan SH (2017) Temporal trends in sperm count: a systematic review and meta-regression analysis. Hum Reprod Update 23(6):646-659

25. Eldamnhoury EM, Elatrash GA, Rashwan HM, El-Sakka AI (2018) Association between leukocytospermia and semen interleukin-6 and tumor necrosis factor-alpha in infertile men. Andrology 6(5):775-780

26. Naito M, Itoh M (2008) Patterns of infiltration of lymphocytes into the testis under normal and pathological conditions in mice. Am J Reprod Immunol 59(1):55-61

27. Zhu R, Wang J, Feng T, Hu X, Jiang C, Wang X, Li K, Sang Y, Hua Y, Sun H, Yao B, Li C (2019) The alteration of RhoA geranylgeranylation and Ras farnesylation breaks the integrity of the blood-testis barrier and results in hypospermatogenesis. Cell Death Dis 10(6):450

28. Liu M, Zhu C, Bai S, Li X, Fu K, Ye L, Zheng K (2018) An in vivo method to study mouse blood-testis barrier integrity. J Vis Exp 142:e58512

29. Condorelli R, Calogero AE, La Vignera S (2013) Relationship between testicular volume and conventional or nonconventional sperm parameters. Int J Endocrinol 2013:145792

30. Aversa A, Bruzziches R, Spera G (2005) Diagnosing erectile dysfunction: the penile dynamic colour duplex ultrasound revisited. Int J Androl 28(2):61-63

31. La Vignera S, Cannarella R, Condorelli RA, Calogero AE (2020) Disorders of puberty: endocrinology of the pre-pubertal testis. J Clin Med 9(3):780

Publisher's Note Springer Nature remains neutral with regard to jurisdictional claims in published maps and institutional affiliations. 